\title{
Self-study of the non-extension sign in an e-learning program improves diagnostic accuracy of invasion depth of early gastric
} cancer

\section{다(1)우우}

\section{Authors}

Minoru Kato' ${ }^{1}$ Noriya Uedo', Takashi Nagahama², Kenshi Yao², Hisashi Doyama ${ }^{3}$, Shigetsugu Tsuji ${ }^{3}$, Takuji Gotoda ${ }^{4}$, Takuji Kawamura $^{5}$, Masahide Ebi ${ }^{6}$, Katsumi Yamamoto ${ }^{7}$, Tomofumi Akasaka ${ }^{8}$, Hajime Takatori ${ }^{9}$, Osamu Handa ${ }^{10}$, Takuji Akamatsu ${ }^{11}$, Jun Nishikawa ${ }^{12}$, Takuto Hikichi ${ }^{13}$, Takeshi Yamashina ${ }^{14}$, Akira Imoto ${ }^{15}$, Yoko Kitamura ${ }^{16}$, Tatsuya Mikami $^{17}$, Tomoyuki Koike ${ }^{18}$, Shuichi Ohara ${ }^{19}$, Shinji Kitamura ${ }^{20}$, Tatsuya Yamaguchi ${ }^{21}$, Tetsu Kinjo ${ }^{22}$, Taro Inoue ${ }^{23}$, Sho Suzuki ${ }^{24}$, Akihiro Kaneko ${ }^{25}$, Kingo Hirasawa ${ }^{26}$, Kyosuke Tanaka ${ }^{27}$, Takahiro Kotachi28, Kazuhiro Miwa ${ }^{29}$, Yosuke Toya $^{30}$, Shoichi Kayaba ${ }^{31}$, Atsushi Ikehata ${ }^{32}$, Shinya Minami ${ }^{33}$, Kazuhiro Mizukami ${ }^{34}$, Hirohisa Oya ${ }^{35}$, Nobuyuki Ara ${ }^{36}$, Yasushi Fukumoto ${ }^{37}$, Takuya Komura ${ }^{38}$, Toshiyuki Yoshio ${ }^{39}$, Ryutaro Morizono ${ }^{40}$, Kenji Yamazaki ${ }^{41}$, Yuichi Shimodate ${ }^{42}$, Kohei Yamanouchi ${ }^{43}$, Noboru Kawata ${ }^{44}$, Masayuki Kumagai ${ }^{45}$, Yoshinori Sato ${ }^{46}$, Kiyotaka Umeki ${ }^{47}$, Daisuke Kawai ${ }^{48}$, Tokuma Tanuma ${ }^{49}$, Maiko Kishino ${ }^{50}$, Jun Konishi ${ }^{51}$, Tetsuya Sumiyoshi ${ }^{52}$, Shohei Oka ${ }^{53}$, Mitsuhiro Kono $^{54}$, Takeshi Sakamoto ${ }^{55}$, Yohei Horikawa ${ }^{56}$, Motoki Ohyauchi ${ }^{57}$, Keiichi Hashiguchi ${ }^{58}$, Yohei Waseda ${ }^{59}$, Toyotaka Kasai60, Hiroyuki Aoyagi ${ }^{61}$, Hirokazu Oyamada ${ }^{62}$, Masakuni Shoji63, Shu Kiyotoki ${ }^{64}$, Sho Asonuma ${ }^{65}$, Shunsuke Orikasa ${ }^{66}$, Chika Akaishi ${ }^{67}$, Yasuaki Nagami ${ }^{68}$, Satoshi Nakata ${ }^{69}$, Fumiyo lida ${ }^{70}$, Tatsuma Nomura ${ }^{71}$, Kei Tominaga ${ }^{72}$, Kohei Oka73, Yoshinori Morita ${ }^{74}$, Haruhisa Suzuki ${ }^{75}$, Keiji Ozeki ${ }^{76}$, Shiko Kuribayashi77, Yoichi Akazawa ${ }^{78}$, Sho Sasaki ${ }^{79}$, Tetsuhiko Mikami ${ }^{80}$, Goro Miki ${ }^{81}$, Tatsushi Sano ${ }^{82}$, Hiro Satoh ${ }^{83}$, Munetaka Nakamura ${ }^{84}$, Wataru Iwai ${ }^{85}$, Hideki Tawa $^{86}$, Masafumi Wada ${ }^{87}$, Daisuke Yoshimura ${ }^{88}$, Yasuhiro Hisanaga ${ }^{89}$, Toshio Shimokawa ${ }^{90}$, Hideki Ishikawa ${ }^{91}$

Institutions

1 Department of Gastrointestinal Oncology, Osaka International Cancer Institute, Osaka, Japan

2 Department of Endoscopy, Fukuoka University Chikushi Hospital, Chikushino, Japan

3 Department of Gastroenterology, Ishikawa Prefectural Central Hospital, Kanazawa, Japan

4 Division of Gastroenterology and Hepatology, Department of Medicine, Nihon University School of Medicine, Tokyo, Japan

5 Department of Gastroenterology, Kyoto Second Red Cross Hospital, Kyoto, Japan

6 Department of Gastroenterology, Aichi Medical University School of Medicine, Nagakute, Japan

7 Department of Gastroenterology, Japan Community Healthcare Organization, Osaka Hospital, Osaka, Japan

8 Department of Gastroenterology and Hepatology, National Hospital Organization Osaka National Hospital, Osaka, Japan

9 Department of gastroenterology, Kanazawa University Hospital, Kanazawa, Japan

10 Department of Molecular Gastroenterology and Hepatology, Graduate School of Medical Science, Kyoto Prefectural University of Medicine, Kyoto, Japan

11 Department of Gastroenterology and Hepatology, Japanese Red Cross Society Wakayama Medical Center, Wakayama, Japan

12 Department of Laboratory Science, Yamaguchi University Graduate School of Medicine, Ube, Japan
13 Department of Endoscopy, Fukushima Medical University Hospital, Fukushima, Japan

14 Department of Gastroenterology and Hepatology, Osaka Red Cross Hospital, Osaka, Japan

15 Second Department of Internal Medicine, Osaka Medical College, Takatsuki, Japan

16 Center for Digestive and Liver Disease, Department of Gastroenterology, Nara City Hospital, Nara, Japan

17 Division of Endoscopy, Hirosaki University Hospital, Hirosaki, Japan

18 Division of Gastroenterology, Tohoku University Graduate School of Medicine, Sendai, Japan

19 Department of Gastroenterology, Tohoku Rosai Hospital, Sendai, Japan

20 Department of Gastroenterology and Oncology, Tokushima University Graduate School of Biomedical Sciences, Tokushima, Japan

21 First Department of Internal Medicine, University of Yamanashi, Yamanashi, Japan

22 Department of Endoscopy, University of the Ryukyus Hospital, Okinawa, Japan

23 Department of Gastroenterology and Hepatology, Kishiwada Tokushukai Hospital, Kishiwada, Japan

24 Department of Gastroenterology and Hepatology, Center for Digestive Disease, and Division of Endoscopy, University of Miyazaki Hospital, Miyazaki, Japan

25 Department of Gastroenterological Medicine, Kobe Rosai Hospital, Kobe, Japan 
26 Division of Endoscopy, Yokohama City University Medical Center, Yokohama, Japan

27 Department of Endoscopy, Mie University Hospital, Tsu, Japan

28 Department of Endoscopy, Hiroshima University Hospital, Hiroshima, Japan

29 Department of Gastroenterology, Komatsu Municipal Hospital, Komatsu, Japan

30 Department of Gastroenterology, Department of Internal Medicine, School of Medicine, Iwate Medical University, Morioka, Japan

31 Department of Gastroenterology, Iwate Prefectural Isawa Hospital, Ohshu, Japan

32 Department of Gastroenterology, Iwate Prefectural Central Hospital, Morioka, Japan

33 Department of Gastroenterology, Oji General Hospital, Tomakomai, Japan

34 Department of Gastroenterology, Oita University, Yufu, Oita, Japan

35 Department of Gastroenterology and Hepatology, Saiseikai Suita Hospital, Suita, Japan

36 Department of Gastroenterology, National Hospital Organization Sendai Medical Hospital, Sendai, Japan

37 Department of Gastroenterology, National Hospital Organization Okayama Medical Center, Okayama, Japan

38 Department of Gastroenterology, National Hospital Organization Kanazawa Medical Center, Kanazawa, Japan

39 Department of Gastroenterology, Cancer Institute Hospital, Tokyo, Japan

40 Section of Gastroenterology, Department of Internal Medicine, Kin-ikyo Chuo Hospital, Sapporo, Japan

41 Department of Gastroenterology, Gifu Prefectural General Medical Center, Gifu, Japan

42 Department of Gastroenterology and Hepatology, Kurashiki Central Hospital, Kurashiki, Japan

43 Department of Gastroenterology, Takagi Hospital, Okawa, Japan

44 Division of Endoscopy, Shizuoka Cancer Center, Nagaizumi-cho Sunto-gun, Japan

45 Department of Gastroenterology, Yokkaichi Municipal Hospital, Yokkaichi, Japan

46 Division of Gastroenterology and Hepatology, Department of Internal Medicine, St. Marianna University School of Medicine, Kawasaki, Japan

47 Department of Gastroenterology, Chiba-Nishi Genera Hospital, Matsudo, Japan

48 Department of Gastroenterology, Tsuyama Chuo Hospital, Tsuyama, Japan

49 Center for Gastroenterology, Teine-Keijinkai Hospital, Sapporo, Japan

50 Department of Gastroenterology, Tokyo Women's Medical University Hospital, Tokyo, Japan

51 Department of Gastroenterology, Tochigi Cancer Center, Utsunomiya, Japan
52 Department of Gastroenterology, Tonan Hospital, Sapporo, Japan

53 Department of Gastroenterology and Hepatology, Okayama University Graduate School of Medicine, Dentistry, and Pharmaceutical Sciences, Okayama, Japan

54 Department of Gastroenterology, Higashisumiyoshi Morimoto Hospital, Osaka, Japan

55 Department of Gastroenterological Oncology, Hyogo Cancer Center, Akashi, Japan

56 Department of Gastroenterology, Hiraka General Hospital, Yokote, Japan

57 Department of Gastroenterology, Osaki Citizen Hospital, Osaki, Japan

58 Department of Endoscopy, Nagasaki University Hospital, Nagasaki, Japan

59 Department of Internal Medicine, Asanogawa General Hospital, Kanazawa, Japan

60 Department of Gastroenterology, Fukaya Red Cross Hospital, Saitama, Japan

61 Department of Gastroenterology, Fukui Prefectural Hospital, Fukui, Japan

62 Department of Gastroenterology, Matsushita Memorial Hospital, Moriguchi, Japan

63 Department of Gastroenterology, Faculty of Medicine, Yamagata University, Yamagata, Japan

64 Department of Gastroenterology, Shuto General Hospital, Yanai, Japan

65 Department of Gastroenterology, South Miyagi Medical Center, Shibata-gun, Japan

66 Department of Gastroenterology, Iwate Prefectural Kuji Hospital, Kuji, Japan

67 Department of Gastroenterology, Iwate Prefectural Chubu Hospital, Kitakami, Japan

68 Department of Gastroenterology, Osaka City University Graduate School of Medicine, Osaka, Japan

69 Department of Gastroenterology, Rakuwakai Otowa Hospital, Kyoto, Japan

70 Department of Gastroenterology, Japan Community Healthcare Organization, Kanazawa Hospital, Kanazawa, Japan

71 Department of Gastroenterology, Kinan Hospital, Minamimuro, Mie, Japan

72 Department of Gastroenterology, Kaga Medical Center, Kaga, Japan

73 Department of Gastroenterology and Hepatology, North Medical Center, Kyoto Prefectural University of Medicine, Yosa, Japan

74 Division of Gastroenterology, Department of Internal Medicine, Kobe University Graduate School of Medicine, Kobe, Japan

75 Endoscopy Division, National Cancer Center Hospital, Tokyo, Japan

76 Department of Gastroenterology and Metabolism, Nagoya City University Graduate School of Medical Sciences, Nagoya, Japan 
77 Clinical Investigation and Research Unit, Gunma University Hospital, Maebashi, Japan

78 Department of Gastroenterology, Juntendo University, School of Medicine, Tokyo, Japan

79 Department of Gastroenterology, Tokuyama Central Hospital, Shunan, Japan

80 Department of Gastroenterology, Towada City Hospital, Towada, Japan

81 Department of Gastroenterology, Nobeoka Medical Association Hospital, Nobeoka, Japan

82 Department of Gastroenterology, Moriguchi Keijinkai Hospital, Moriguchi, Japan

83 Department of Gastroenterology, Midori Municipal Hospital, Nagoya, Japan

84 Department of Gastroenterology, Hagi Civil Hospital, Hagi, Japan

85 Department of Gastroenterology, Miyagi Cancer Center, Natori, Japan

86 Department of Gastroenterology, Seikeikai Hospital, Sakai, Japan

87 Department of Gastroenterology, National Hospital Organization Kyushu Medical Center, Fukuoka, Japan

88 Division of Gastroenterology, Saiseikai Fukuoka General Hospital, Fukuoka, Japan

89 Department of Gastroenterology, Ogaki Municipal Hospital, Ogaki, Japan

90 Department of Clinical Study Support Center, Wakayama Medical University, Wakayama, Japan

91 Department of Molecular-Targeting Cancer Prevention, Kyoto Prefectural University of Medicine, Kyoto, Japan

submitted 10.11.2018

accepted after revision 1.4 .2019

Bibliography

DOI https://doi.org/10.1055/a-0902-4467 |

Endoscopy International Open 2019; 07: E871-E882

(c) Georg Thieme Verlag KG Stuttgart · New York eISSN 2196-9736
Corresponding author

Noriya Uedo, MD, Department of Gastrointestinal

Oncology, Osaka International Cancer Institute, 3-1-69

Otemae Chuo-ku, Osaka 541-8567, Japan

Fax: +81-6-6945-1900

noriya.uedo@gmail.com

\section{ABSTRACT}

Background and study aims We developed an e-learning program for endoscopic diagnosis of invasion depth of early gastric cancer (EGC) using a simple diagnostic criterion called non-extension sign, and the contribution of selfstudy quizzes to improvement of diagnostic accuracy was evaluated.

Methods We conducted a prospective randomized controlled study that recruited endoscopists throughout Japan. After completing a pretest, the participants watched video lectures and undertook post-test 1 . The participants were then randomly allocated to either the self-study or nonself-study group, and participants in the first group completed the self-study program that comprised 100-case quizzes. Finally, participants in both groups undertook post-test 2 . The primary endpoint was the difference in post-test 2 scores between the groups. The perfect score for the tests was set as 100 points.

Results A total of 423 endoscopists completed the pretest and were enrolled. Post-test 1 was completed by 415 endoscopists and 208 were allocated to the self-study group and 207 to the non-self-study group. Two hundred and four in the self-study group and 205 in the non-selfstudy group were included in the analysis. Video lectures improved the mean score of post-test 1 from 72 to 77 points. Participants who completed the self-study quizzes showed significantly better post-test 2 scores compared with the non-self-study group (80 vs. 76 points, respectively, $P<0.0001)$.

Conclusions Our e-learning program showed that selfstudy quizzes consolidated knowledge of the non-extension sign and improved diagnostic ability of endoscopists for invasion depth of EGC.

\section{Introduction}

Accurate endoscopic diagnosis of invasion depth of early gastric cancer (EGC) is indispensable for treatment decision-making. In the current Japanese gastric cancer treatment guidelines, endoscopic resection, such as endoscopic mucosal resection (EMR) or endoscopic submucosal dissection (ESD), is indicated as standard treatment for patients with clinically intramucosal (T1a) carcinoma. Resection can be regarded as curative for histologically $\mathrm{T} 1 \mathrm{a}$ or shallow $(<500 \mu \mathrm{m}$ from the muscularis muco- sae) submucosal carcinoma (T1b1), whereas surgery is required for deep ( $\geq 500 \mu \mathrm{m}$ from the muscularis mucosae) submucosal carcinoma (T1b2) [1]. Therefore, prediction of T1a-T1b1 and T1b2 carcinoma is of particular importance for determining whether to perform EMR/ESD or surgery.

Although the usefulness of endoscopic ultrasonography (EUS) is reported, conventional white light endoscopy (WLE) is commonly used to predict invasion depth of EGC in clinical practice. One of the problems in acquiring the requisite skill for diagnosis of invasion depth of EGC using WLE is that the various findings suggestive of submucosal cancer are subjective 

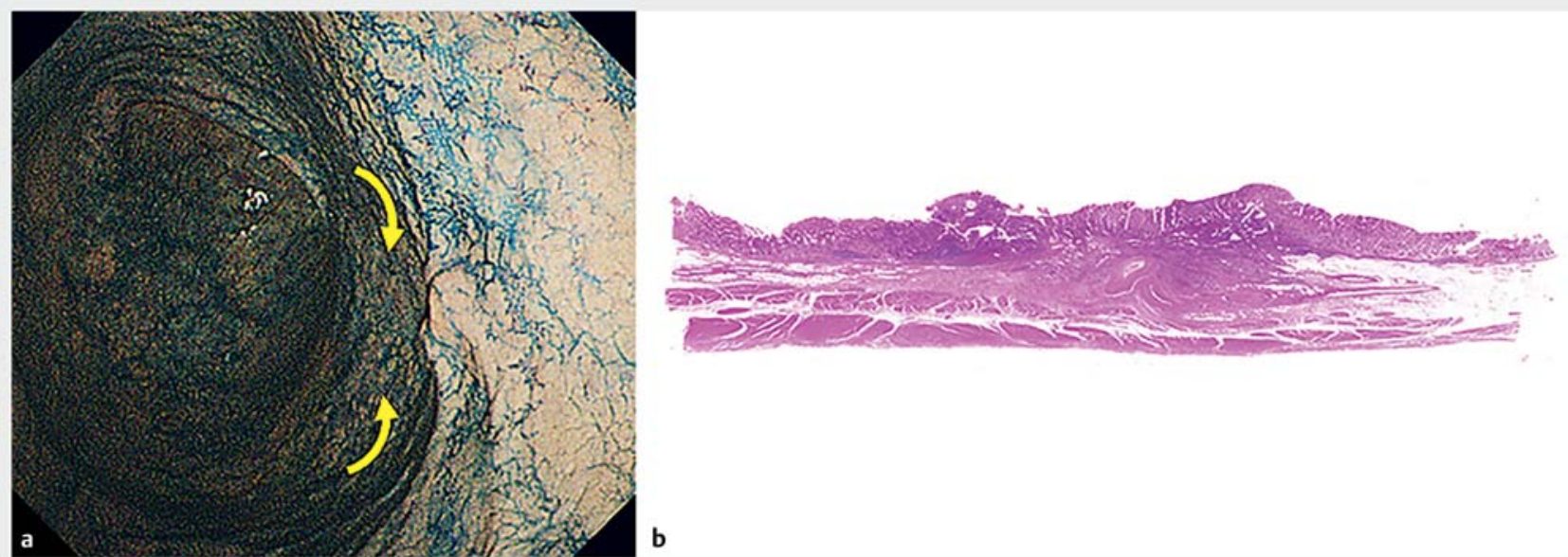

Fig. 1 a A representative endoscopic image of early gastric cancer with deep submucosal invasion (T1b2) showing the non-extension sign. The strongly distended gastric wall was gradually elevated toward the submucosa-invasive area (yellow arrows), which had a trapezoid appearance. $\mathbf{b}$ This endoscopic finding was well-supported by histological findings of the resected specimen. Invasion depth was $1500 \mu \mathrm{m}$ from the muscularis mucosae in this case.

and the most significant finding is uncertain. To overcome this problem, Nagahama et al. proposed one simple diagnostic criterion called the non-extension sign, which had an accuracy of $96.9 \%$ for defining T1b2 carcinoma [2]. The non-extension sign is a phenomenon in which the submucosal invasive area and surrounding mucosa protrude into the lumen, resembling a trapezoidal shape, when the gastric wall is well distended by endoscopic air insufflation ( $\boldsymbol{F}$ Fig. 1 ). Unlike the other diagnostic findings of the tumor depth, such as tumor diameter, macroscopic type, and coloration, which were mainly brought by statistical analyses, the non-extension sign has a direct causal relationship with the histopathological feature of deep submucosal invasion. In addition, because assessment of the non-extension sign is always done under the same observational conditions (i. e., sufficient extension of the gastric wall with the air), we can judge presence of the sign rather objectively. Dissemination of knowledge about this diagnostic criterion may improve diagnostic accuracy of endoscopists. Experience is another major issue in acquisition of reliable diagnostic skill. In fact, many endoscopists in low-volume hospitals have limited opportunities to see EGC patients. We hypothesized that, especially for diagnosing invasion depth of EGC by endoscopic appearance, accumulation of experience is important to achieve sufficient diagnostic skill as well as obtaining knowledge of the simplified diagnostic criterion.

We developed an e-learning program that consisted of webbased video lectures and self-study quizzes in which endoscopists could learn the concept of the non-extension sign, and accumulate experience in diagnosing invasion depth of EGC using the non-extension sign. The aim of this study was to investigate whether this accumulation of experience independently contributes to improvement in endoscopists' ability to diagnose invasion depth of EGC.

\section{Methods}

\section{Study design}

We conducted a prospective randomized controlled trial to assess the effectiveness of the e-learning program. This study was designed according to the Consolidated Standards of Reporting Trials (CONSORT) 2010 Statement. The study protocol was approved by the Institutional Review Board of Osaka International Cancer Institute, Japan (No. 1605276051, dated July 6, 2016). The study was registered on July 28, 2017 in the University Hospital Medical Network Clinical Trials Registry (UMIN-CTR), which is accepted as a primary registry by the International Committee of Medical Journal Editors (ICMJE) since 2007 (UMIN000028413). Written informed consent was obtained from all participating gastrointestinal endoscopists.

\section{Participants}

We recruited gastrointestinal endoscopists from all over Japan between August and November 2017. Inclusion criteria were physicians who engaged in upper gastrointestinal endoscopy and provided consent to participate in the study. Before starting the e-learning program, we collected details of the following characteristics of the participants: age, sex, type of affiliated institutions, years of experience in upper gastrointestinal endoscopy, amount of experience in gastric ESD, and annual numbers of gastric ESDs performed in their affiliated institutions. We created a website where participants were able to take the diagnostic tests and view video lectures and the selfstudy program. Each participant received an e-mail from a data center with his or her personal login username and password for the website. The data center monitored participants' activity on the website and collected the test results via the Internet. When the deadline for the tasks on the website was nearing, a reminder e-mail was sent to each participant who had not completed the tasks. 


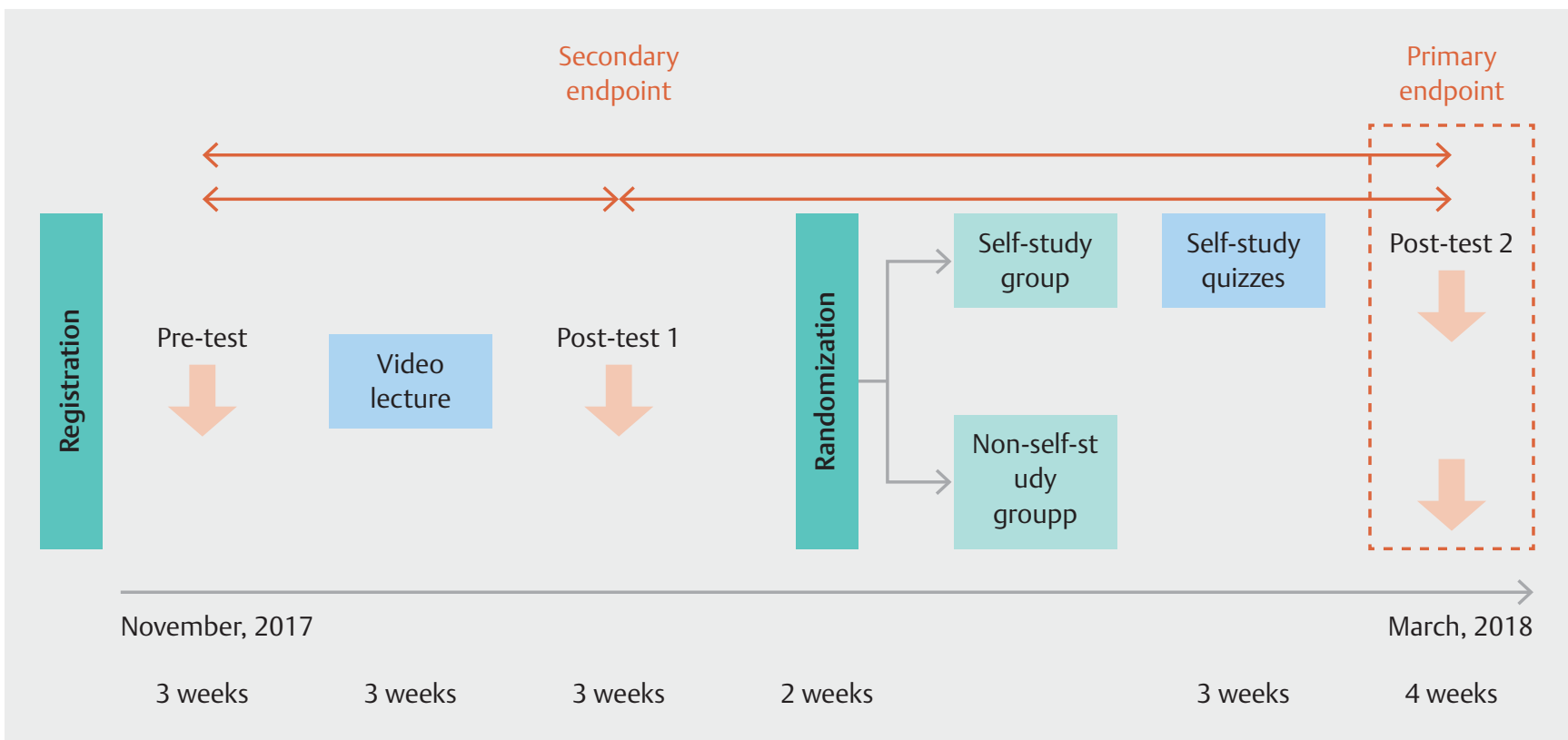

- Fig. 2 Study overview. The primary endpoint was the difference in scores for post-test 2 between the self-study and non-self-study groups. Secondary endpoints were differences in scores between the pretest and post-test 1, post-test 1 and post-test 2 , and the pretest and post-test 2 in each group.

\section{Interventions}

The participants were asked to take the diagnostic test (pretest) on the website between 9:00 a.m. November 21, 2017 and 8:59 a.m. December 12, 2017. Then, they were told to watch the video lecture via the Internet between 9:00 a.m. December 12, 2017 and 8:59 a.m. January 5, 2018 (video lecture period). After the video lecture period, between 9:00 a.m. January 5, 2018 and 8:59 a.m. January 26, 2018, the participants took the second diagnostic test (post-test 1). After post-test 1 , participants were randomly assigned to the self-study or non-self-study group. Participants in the self-study group were allowed to access the self-study program on the website between 9:00 a.m. February 9, 2018 and 8:59 a.m. March 2, 2018 (self-study period), whereas access to the self-study program was blocked for those in the non-self-study group. Afterwards, participants in both groups undertook another diagnostic test (post-test 2) between 9:00 a.m. March 2, 2018 and 8:59 a.m. March 30, 2018 ( $\triangleright$ Fig.2). Finally, after completing posttest 2 , the self-study program was opened for all study participants to freely access and learn the content.

\section{Diagnostic tests (pretest, post-test 1 and post-test 2)}

The diagnostic test comprised endoscopic images of 40 nonconsecutive cases of histologically proven EGC ( $\triangleright$ Table 1$)$. We used the same 40 cases (20 T1a-T1b1 lesions without the nonextension sign and $20 \mathrm{~T} 1 \mathrm{~b} 2$ lesions with the non-extension sign) in the same order for the pretest, post-test 1 and posttest 2; however, this information was not disclosed to the participants. All endoscopic images were taken during actual clinical practice by an expert endoscopist (T.N.) at Fukuoka University
Chikushi Hospital using a high-definition videoendoscopy system (EVIS LUCERA Spectrum System; Olympus CO, Tokyo, Japan) and videogastroscopes (GF-Q240, GIF-Q240Z, GIF-H260 or GIF-H260Z; Olympus CO). We chose two endoscopic images for each EGC case. At least one of the images had sufficient quality to judge the non-extension sign ( $\mathbf{F i g . 3}$ ), that is, an endoscopic image of the EGC observed from an oblique position under the gastric wall sufficiently extended by air insufflation [2].

The participants accessed the diagnostic test on the website via the Internet using their own username and password. In the test, participants were asked to diagnose whether the EGC presented was T1a-T1b1 or T1b2 carcinoma. For each case, they also were asked about presence or absence of the following findings, which had been reported as the signs of T1b2 carcinoma [2-8]: 1) large tumor size (>30 $\mathrm{mm}$ in diameter); 2) remarkable redness; 3 ) enlargement of the converging folds; 4) margin elevation; 5) irregular surface; and 6) non-extension sign. Presence or absence of each of the features in the target EGCs was preliminarily determined by the expert endoscopists (T.N. and N.U.), who had experience in diagnosing EGC invasion depth in more than 1000 cases ( $\triangleright$ Table 1 ). Once an answer for each question was selected, the question could no longer be accessed. Test scores were marked for correct answers of T1aT1b1 or T1b2 up to a total of 100 points. As for the assessment of aforementioned 6 endoscopic findings, participant's judgement was regarded as correct if it was matched with the predetermined assessment by the experts (T.N. and N.U.). The correct answers were not revealed until all the participants had completed post-test 2. 
- Table 1 Characteristics of early gastric cancers used in the tests.

\begin{tabular}{|c|c|c|}
\hline & T1a-T1b1 & T1b2 \\
\hline & $\mathbf{n}=\mathbf{2 0}$ & $\mathbf{n}=\mathbf{2 0}$ \\
\hline Median size, (range), mm & $20(10-32)$ & $18(8-48)$ \\
\hline \multicolumn{3}{|l|}{ Location, n } \\
\hline - Upper & 1 & 1 \\
\hline - Middle/Lower & 19 & 19 \\
\hline \multicolumn{3}{|l|}{ Macroscopic appearance, $n$} \\
\hline - Elevated & 4 & 3 \\
\hline - Depressed & 13 & 15 \\
\hline - Mixed & 3 & 2 \\
\hline \multicolumn{3}{|l|}{ Ulcer or scar, n } \\
\hline - Present & 11 & 12 \\
\hline - Absent & 9 & 8 \\
\hline \multicolumn{3}{|l|}{ Histological type, $\mathrm{n}$} \\
\hline - Differentiated & 17 & 11 \\
\hline - Undifferentiated & 3 & 9 \\
\hline \multicolumn{3}{|c|}{ Invasion depth from the muscularis mucosae, $\mathrm{n}$} \\
\hline . $500-999 \mu \mathrm{m}$ & $\mathrm{N} / \mathrm{A}$ & 5 \\
\hline - $\geq 1000 \mu \mathrm{m}$ & $\mathrm{N} / \mathrm{A}$ & 15 \\
\hline \multicolumn{3}{|l|}{ Endoscopic findings, $\mathrm{n}$} \\
\hline - Large tumor size (>30 mm) & 1 & 2 \\
\hline - Remarkable redness & 4 & 8 \\
\hline - Enlargement of the converging folds & 0 & 0 \\
\hline - Margin elevation & 7 & 12 \\
\hline - Irregular surface & 8 & 10 \\
\hline - Non-extension sign & 0 & 20 \\
\hline
\end{tabular}
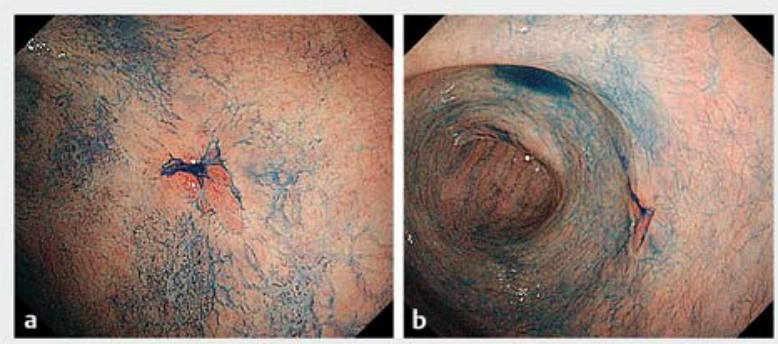

- Fig. 3 An example case of EGC used in the diagnostic tests. Two endoscopic images are shown for one case. This case was EGC with deep submucosal invasion (T1b2). a Endoscopic images showing the lesion in en-face view and $\mathbf{b}$ oblique view are presented. Photograph $\mathbf{b}$ has sufficient quality to judge the non-extension sign because the oblique view is a more accurate condition for diagnosis than en-face view. EGC, early gastric cancer.
- Table 2 Characteristics of early gastric cancers used in the selfstudy quizzes.

\begin{tabular}{|l|c|c|}
\hline & T1a-T1b1 & T1b2 \\
\hline & $\mathbf{n = 5 5}$ & $\mathbf{n = 4 5}$ \\
\hline Median size, (range), mm & $24(6-70)$ & $22(6-120)$ \\
\hline Location, $\mathrm{n}(\%)$ & & \\
\hline - Upper & $6(11)$ & $8(18)$ \\
\hline - Middle/lower & $49(89)$ & $37(82)$ \\
\hline Macroscopic appearance, $\mathrm{n}(\%)$ & & \\
\hline - Elevated & $25(45)$ & $19(42)$ \\
\hline - Depressed & $30(55)$ & $11(24)$ \\
\hline - Mixed & $0(0)$ & $15(33)$ \\
\hline Ulcer or scar, $\mathrm{n}$ (\%) & & \\
\hline - Present & $20(36)$ & $12(27)$ \\
\hline - Absent & $35(64)$ & $33(73)$ \\
\hline Histological type, $\mathrm{n}(\%)$ & & \\
\hline - Differentiated & $47(85)$ & $31(69)$ \\
\hline - Undifferentiated & $8(15)$ & $14(31)$ \\
\hline Endoscopic findings, $\mathrm{n}(\%)$ & & \\
\hline - Large tumor size (>30 mm) & $19(35)$ & $11(24)$ \\
\hline - Remarkable redness & $4(7.3)$ & $16(36)$ \\
\hline - Enlargement of the converging folds & $0(0)$ & $3(6.7)$ \\
\hline - Margin elevation & $5(9.1)$ & $25(56)$ \\
\hline - Irregular surface & $4(7.3)$ & $20(44)$ \\
\hline
\end{tabular}

\section{Video lecture}

The video lecture was given in Japanese by a doctor (T.N.), who first reported usefulness of the non-extension sign [2]. The video lecture was recorded by adding voice narrations to PowerPoint slides (Microsoft Corporation, Redmond, Washington, United States). The lecture was composed of two chapters. In the first chapter, the principle and basics of the non-extension sign were explained, and in the second chapter, the actual endoscopic techniques for capturing and assessing the endoscopic images and pointers for accurate diagnosis of the nonextension sign were explained. Each lecture was approximately 30 minutes. All participants were required to watch these webbased lecture videos at least once, and thereafter, as many times as they wanted until the end of the video lecture period.

\section{Self-study quizzes}

Self-study quizzes consisted of endoscopic images of 100 cases of EGC ( $\triangleright$ Table 2). A single representative endoscopic image was prepared for each lesion. The invasion depth of all lesions used in the self-study quizzes had been histologically confirmed as either T1a-T1b1 or T1b2. The 100 endoscopic images used in 


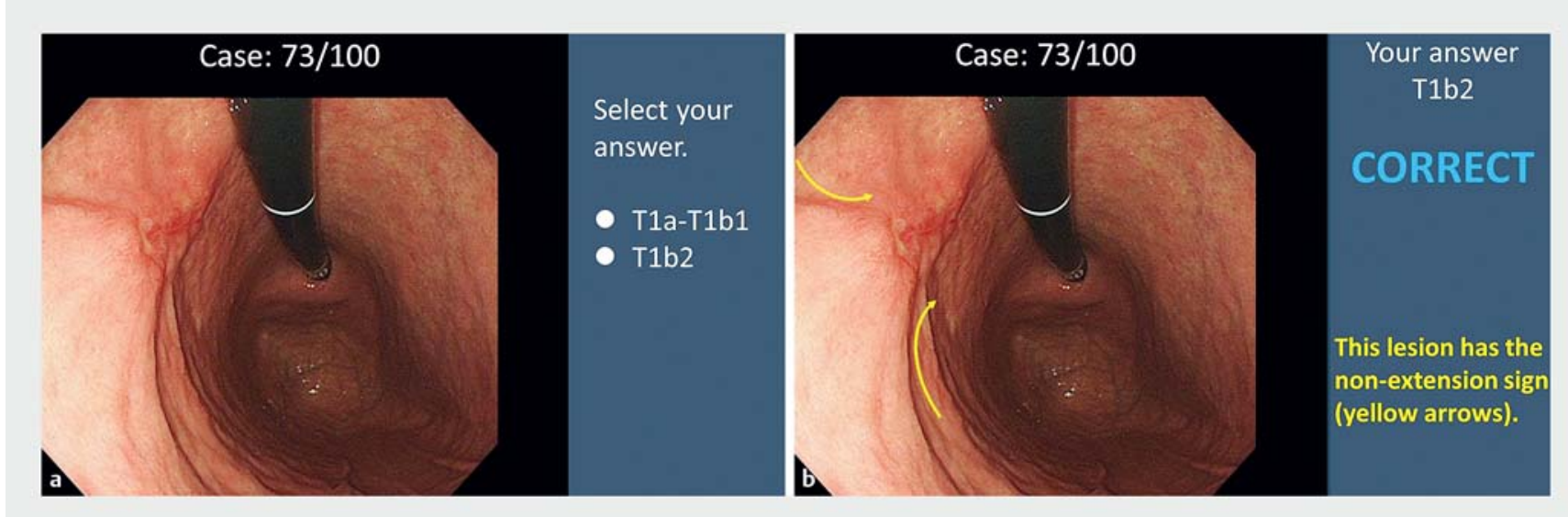

- Fig.4 An example image of EGC with deep submucosal invasion (T1b2) used in the self-study quizzes. The quiz question was translated into English for this manuscript. a The first slide shows one EGC image with a question asking the user to select whether the invasion depth was T1a-T1b1 or T1b2. b The second slide appeared immediately after a participant clicked the answer and showed whether the answer was correct or incorrect. If the lesion was positive for the non-extension sign, yellow arrows indicated the position of the sign in the same endoscopic image on the second slide. EGC, early gastric cancer.

the self-study quizzes were taken by expert endoscopists at Fukuoka University Chikushi Hospital (T.N.) and Osaka International Cancer Institute (N.U.) under the same conditions as the diagnostic test images. There was no overlap of images between the self-study quizzes and the diagnostic tests. The 100 cases for the self-study quizzes consisted of 55 cases of T1a-T1b1 carcinoma and 45 of T1b2 carcinoma that were arranged randomly. Each case had a pair of slides of the endoscopic images, and in the first slide, the participants were asked to answer whether the invasion depth was T1a-T1b1 or T1b2 ( $\vee$ Fig.4a). Then, after clicking the answer button for either T1a-T1b1 or T1b2 on the first slide, the second slide immediately showed whether the answer was correct or incorrect ( $\triangleright$ Fig.4b). In addition, if the lesion was positive for the non-extension sign, the site of the non-extension sign was indicated in the same endoscopic image on the second slide ( $\triangleright$ Fig. $4 \mathbf{b}$ ). All 100 quizzes in the self-study were required to be completed at least once. The self-study quizzes were open until the end of the self-study period and the participants were allowed to repeat the quizzes as many times as they wanted. The number of times that the self-study was undertaken by each participant was recorded.

\section{Outcomes}

The primary endpoint was the difference in the scores for posttest 2 between the self-study and non-self-study groups. Secondary endpoints were differences in the scores between the pretest and post-test 1 , post-test 1 and post-test 2 , and the pretest and post-test 2 in each group ( $\triangleright$ Fig. 2 ). In post-test 2 , sensitivity and specificity of the participants' diagnosis of T1b2 carcinoma were compared between the self-study and the nonself-study groups. The correct judgement rates for presence/ absence of the six endoscopic signs suggesting T1b2 carcinoma (i. e., large tumor size, remarkable redness, enlargement of the converging folds, margin elevation, irregular surface, and the non-extension sign) in post-test 2 were also compared between the self-study and non-self-study groups.

For the self-study group, we compared: 1) scores of posttest 2 between participants who completed the self-study quizzes more than twice and those who completed only once; and 2 ) changes in scores between the pretest and post-test 2 among less experienced and experienced endoscopists. Experience of endoscopy was divided into less experienced ( $<7$ years) and experienced ( $\geq 7$ years) groups based on median years of experience in upper gastrointestinal endoscopy (6 years) in this study cohort.

We divided the $20 \mathrm{~T} 1 \mathrm{~b} 2$ lesions used in the diagnostic test into two subgroups according to submucosal invasion depth (i.e., 5 lesions for $500-999 \mu \mathrm{m}$ group and 15 lesions for $\geq 1000 \mu \mathrm{m}$ group, $>$ Table $\mathbf{1}$ ), and compared the correct answer rate of self-studied participants among the two subgroups in post-test 2.

\section{Sample size calculation}

A preliminary study conducted by five evaluators in the Osaka International Cancer Institute who had knowledge of the nonextension sign but had little diagnostic experience using the non-extension sign in clinical practice showed a mean score of around 80 points for the test used in this study. Therefore, we assumed that the mean score for post-test 2 in the non-selfstudy group would be 80 points and that self-study would improve it to 85 points. We assumed that dispersion of the participants' score results might be rather large, as we intended to recruit endoscopists with varied experience levels. We therefore estimated the standard deviation at 18. Power analysis indicated that more than 410 participants were needed in all, assuming a $5 \%$ significance level and statistical power of $80 \%$ using a two-sided equivalence. 


\section{Randomization}

The randomization sequence was generated by computer software in the data center, and randomized allocation was performed by the doctor (H.I.) at the data center, who was not blinded to allocation. Stratified permuted block randomization was used, with the participants' post-test 1 score and years of experience in upper gastrointestinal endoscopy as pre-adjustment factors. The physician who performed randomized allocation did not know the participants' background information and the allocation was performed automatically based on the above-mentioned stratification rule, which did not cause any bias for randomized allocation.

\section{Statistical analysis}

All analyses were performed using JMP Pro version 13 (SAS Institute, Cary, North Carolina, United States). Scores for posttest 2 in the two groups were compared using unpaired Student's $t$-test. Paired Student's $t$-test was used to compare scores for the diagnostic tests in each group. Multiple comparisons were corrected using Bonferroni's method. Mean sensitivity and specificity of the participants' diagnosis of T1b2 carcinoma in the two groups were compared using unpaired Student's $t$-test. Mean correct judgement rates of the 6 endoscopic signs were compared using unpaired Student's $t$-test. Unpaired Student's $t$-test was used to compare the post-test 2 scores between the participants who undertook the self-training program more than twice and those who undertook it only once. Comparison of the score differences between the pretest and post-test 2 among less experienced and experienced endoscopists in the self-study group was performed by using unpaired Student's t-test. Mean correct T-staging rates between $500-999 \mu \mathrm{m}$ group and $\geq 1000 \mu \mathrm{m}$ group were compared by using unpaired Student's t-test. $P<0.05$ was considered to be statistically significant.

\section{Results}

\section{Participant flow and characteristics: recruitment, baseline data, numbers analyzed}

Among 439 endoscopists assessed for eligibility, 423 from 93 institutions completed the pretest and were included in this study. Of these, 417 participants viewed the video lectures. Post-test 1 was completed by 415 participants, and 208 were allocated to the self-study group and 207 to the non-self-study group. After allocation, 204 participants in the self-study group completed the self-study quizzes and post-test 2, and 205 participants in the non-self-study group completed post-test 2 ( Fig.5). Thirty participants who missed the deadline for each study period were excluded from the analysis and data from 204 participants in the self-study group and 205 in the nonself-study group were analyzed. The groups were well balanced with regard to baseline characteristics ( $>$ Table 3 ).

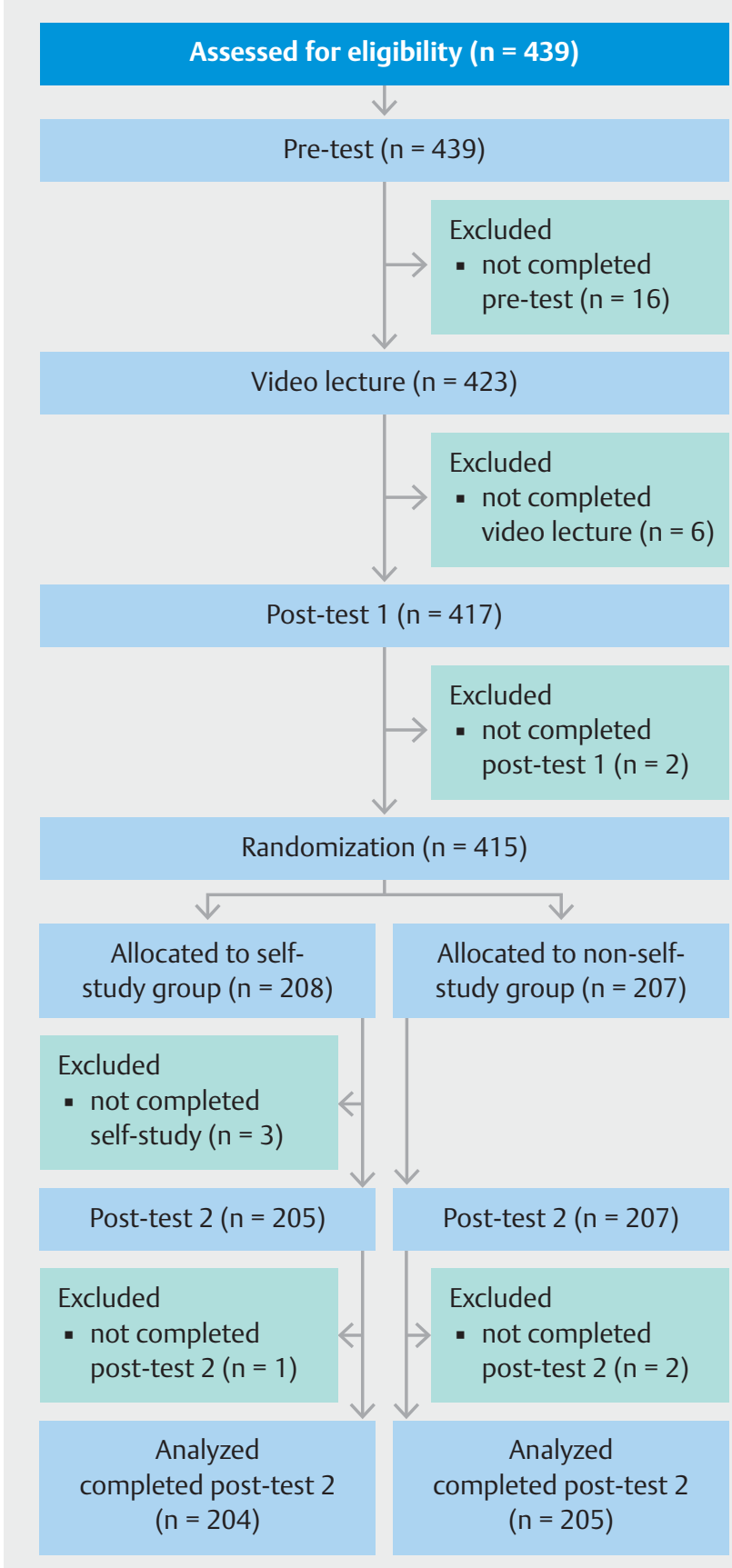

Fig. 5 Participant flow.

\section{Study outcomes}

The mean $[95 \%$ confidence interval $(\mathrm{Cl})$ ] post-test 2 score was significantly higher in the self-study group (80.3 [79.3-81.3]) than in the non-self-study group (75.8 [74.5-77.0], $P<$ 0.0001 , Table 4]. This indicated that self-study subsequent to the video lectures improved knowledge for diagnosis of invasion depth of EGC compared with viewing the video lecture alone.

In both the self-study and non-self-study groups, mean (95\% $\mathrm{Cl}$ ) post-test 1 score was significantly improved by the video 
- Table 3 Characteristics of 409 participants.

\begin{tabular}{|l|c|c|}
\hline & Self-study group & Non-self-study group \\
\hline Age, $\mathbf{n}$ & $\mathbf{n = 2 0 4}$ & $\mathbf{n = 2 0 5}$ \\
\hline - $<35$ years & 111 & \\
\hline - $\geq 35$ years & 93 & 90 \\
\hline Gender, $\mathrm{n}$ & & \\
\hline - Male & 177 & 175 \\
\hline - Female & 27 & 30 \\
\hline Years of experience in upper gastrointestinal endoscopy, $\mathrm{n}$ \\
\hline - $<7$ & 107 & 103 \\
\hline - $\geq 7$ & 97 & 102 \\
\hline Numbers of experience in gastric ESD, $\mathrm{n}$ & \\
\hline - $<30$ & 106 & 103 \\
\hline - $\geq 30$ & 98 & 102 \\
\hline Annual numbers of gastric ESD in affiliated institutions, $\mathrm{n}$ \\
\hline - $<100$ & 73 & 75 \\
\hline - $\geq 100$ & 131 & 100 \\
\hline
\end{tabular}

ESD, endoscopic submucosal dissection

lecture (72.1 [70.9-73.4] to 77.4 [76.2-78.5], $P<0.0001$; and 71.4 [70.3 - 72.6] to 77.1 [76.0 - 78.3], $P<0.0001$, respectively, - Fig.6). Mean post-test 2 score was significantly improved after completing the self-study (77.4 [76.2-78.5] to 80.3 [79.3-81.3], $P<0.0001$, Fig.6), whereas it was significantly reduced in the non-self-study group (77.1 [76.0 - 78.3] to 75.8 [74.5-77.0], $P=0.012$, $\vee$ Fig. 6). This suggested that self-study consolidated the participants' knowledge acquired from the video lecture.

In post-test 2 , the mean $(95 \% \mathrm{Cl})$ sensitivity and specificity for diagnosis of T1b2 carcinoma were $80.3 \%$ (78.6\%-82.0\%) and $80.3 \%(78.2 \%-82.4 \%)$ in the self-study group, and $65.8 \%$ $(63.3 \%-68.3 \%)$ and $85.8 \%(83.8 \%-87.7 \%)$ in the non-selfstudy group ( $\vee$ Table 4 ). Sensitivity (i.e., the ability to correctly detect T1b2 carcinoma) was effectively improved by the selfstudy quizzes $(\mathrm{P}<0.0001)$.

Among the six endoscopic findings indicating T1b2 carcinoma, the non-extension sign was the only one where the mean $(95 \% \mathrm{Cl})$ correct judgement rate was significantly higher in the self-study group $(79.3 \%[78.1 \%-80.4 \%])$ than in the non-selfstudy group (73.1\% [71.9\% - 74.3\%], $P<0.0001$, $>$ Table 4$)$.

In the self-study group, 30 of 204 (14.7\%) participants completed the self-study quizzes more than twice. The mean (95\% $\mathrm{Cl}$ ) post-test 2 score was significantly higher in those who undertook the self-study quizzes more than twice (83.0 [80.3$85.7])$ compared with the remaining 174 participants who undertook the quizzes only once (79.9 [78.8-81.0]) $(P=0.0336)$. Besides, in the self-study group, the mean $(95 \% \mathrm{Cl})$ values of improvement of the scores from the pretest to post-test 2 were significantly higher in less experienced endoscopists than in experienced endoscopists $(\Delta 9.44$ [7.56-11.3] versus $\Delta 6.80$ [4.83-8.78], $P=0.029$ ).

Among the $20 \mathrm{~T} 1 \mathrm{~b} 2$ cases used in the diagnostic test (posttest 2), the 500-999- $\mu \mathrm{m}$ group had significantly lower correct T-staging rates than the $\geq 1000 \mu \mathrm{m}$ group $(61.4 \%[95 \% \mathrm{Cl}$, $45.7-77.1$ ] versus $86.8 \%$ [ $95 \% \mathrm{Cl}, 77.7-95.9$ ], $P=0.0088$, [results of the self-study group]).

\section{Discussion}

In this randomized controlled study, we demonstrated that diagnostic experience accumulated by the self-study quizzes significantly improved the diagnostic ability of endoscopists for in-

- Table 4 Summaries of primary and secondary outcomes.

\begin{tabular}{|c|c|c|c|}
\hline & Self-study group & Non-self-study group & $P$ value \\
\hline Scores of the post-test 2 (Diagnostic accuracy for the invasion depth) & $80.3(79.3-81.3)$ & $75.8(74.5-77.0)$ & $<0.0001$ \\
\hline \multicolumn{4}{|l|}{ Proportion of correct judgement for endoscopic appearances } \\
\hline Large (>30 mm) tumor size (\%) & $73.8(72.3-75.3)$ & $73.4(71.9-74.9)$ & 0.723 \\
\hline Remarkable redness (\%) & $77.1(76.1-78.1)$ & $76.4(75.4-77.3)$ & 0.288 \\
\hline Enlargement of the converging folds (\%) & $88.8(87.3-90.3)$ & $87.2(85.6-88.7)$ & 0.143 \\
\hline Margin elevation (\%) & $62.7(61.7-63.7)$ & $62.3(61.3-63.3)$ & 0.641 \\
\hline Irregular surface (\%) & $65.9(64.9-66.8)$ & $66.2(65.2-67.2)$ & 0.639 \\
\hline Non-extension sign (\%) & $79.3(78.1-80.4)$ & $73.1(71.9-74.3)$ & $<0.0001$ \\
\hline \multicolumn{4}{|l|}{ Diagnostic performance for submucosal invasion } \\
\hline Sensitivity (\%) & $80.3(78.6-82.0)$ & $65.8(63.3-68.3)$ & $<0.0001$ \\
\hline Specificity (\%) & $80.3(78.2-82.4)$ & $85.8(83.8-87.7)$ & 0.0002 \\
\hline
\end{tabular}




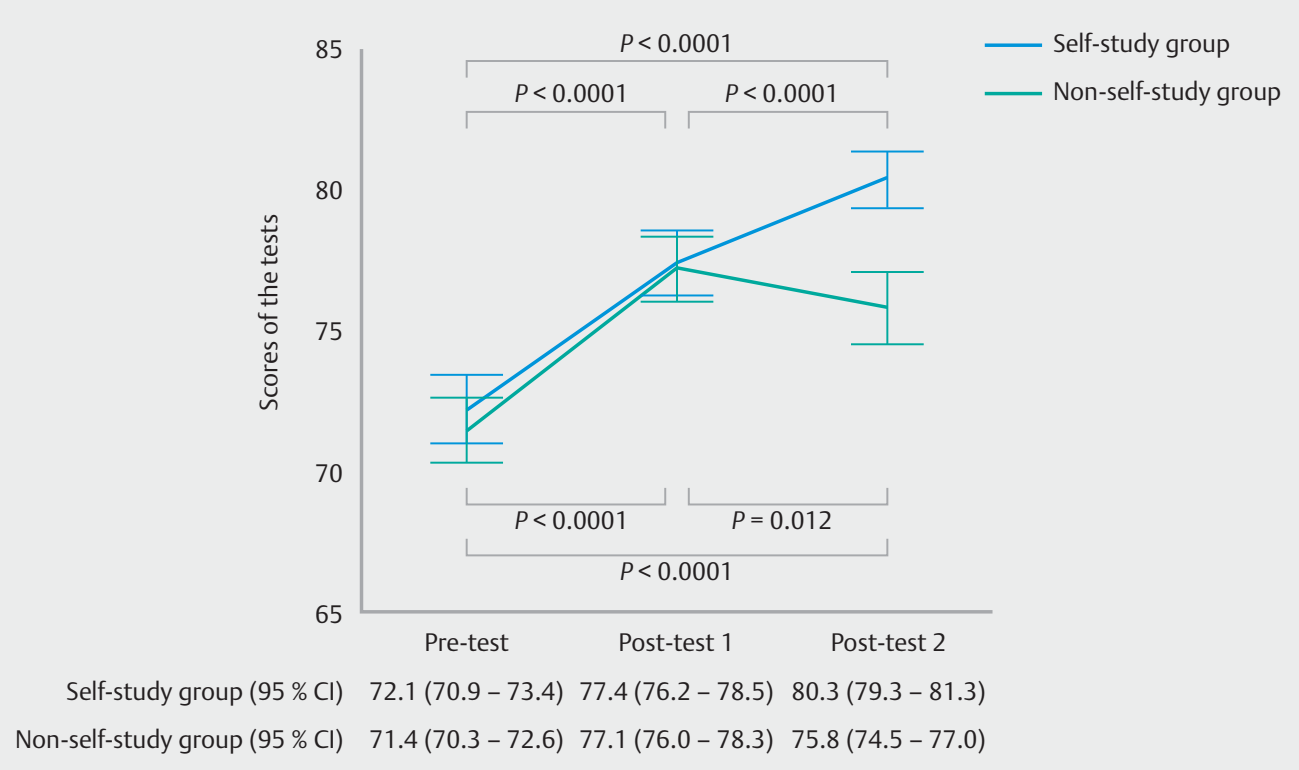

- Fig. 6 Changes in mean scores with $95 \%$ confidence intervals for the pretest, post-test 1 and the post-test 2. P values were for comparisons between two tests. In the self-study and non-self-study groups, mean scores for post-test 2 ( 80.3 and 75.8$)$ and post-test 1 (77.4 and 77.1$)$ were significantly higher than for the pretest $(72.1$ and $71.4 ; P<0.0001)$. In the self-study group, the mean score for post-test 2 (80.3) was significantly higher than for post-test $1(77.4 ; P<0.0001)$. In the non-self-study group, the mean score for post-test $2(75.8)$ was significantly lower than for post-test $1(77.1 ; P=0.012) . P<0.016$ was considered to be statistically significant after correction using Bonferroni's method.

vasion depth of EGC. Although the video lecture significantly improved diagnostic accuracy of the web tests, such a learning effect did not last long without accumulation of experience. In the real world, endoscopists gain knowledge about endoscopy from textbooks, research articles or academic meetings; however, not all endoscopists have sufficient ongoing opportunities to use their knowledge and accumulate experience in their own clinical practice. In this context, our approach of inclusion of content that enables endoscopists to acquire virtual experiences in an e-learning program has substantial value.

Although the non-extension sign was reported to be a reliable marker for determination of tumor depth of EGC, the previous study was conducted by endoscopists in a single institution who were already familiar with using the non-extension sign [2]. Our study, in which $>400$ endoscopists from 93 institutions participated, showed significant improvement in diagnosing invasion depth of EGC following the web-based e-learning program about the non-extension sign. In addition, among the various endoscopic findings suggesting T1 b2 carcinoma, the non-extension sign was the only one where the identification rate became significantly higher by the self-study quizzes. On the other hand, participants' ability to identify other classical endoscopic findings of T1 b2 cancer did not improve even after reviewing many images of EGCs on the self-study material. Because instruction about existence of the non-extension sign was only provided in the self-study quizzes, we can not reach a definitive conclusion, but the abovementioned results may indicate that the non-extension sign can be learned more easily than the other classical endoscopic findings and thus may improve endoscopists' diagnostic ability. We consider that the learning effect of our self-study material was not a result of merely showing many images of EGCs but also the availability of repetitive training on how to identify the non-extension sign.

In this study, diagnostic accuracy of the post-test 2 in the self-study group ( $80.3 \%$ ) was not as high as that in the original study by Nagahama et al (96.9\%) [2]. This might be partly explained by the fact that endoscopic diagnosis was performed real-time in the original study; however, in this study, participants had to estimate invasion depth of EGCs by assessing only two recorded images.

In this study, we elucidated how studying the non-extension sign influenced diagnostic performance in T-staging, not only from the viewpoint of accuracy but also in sensitivity and specificity. More accurate T-staging in the self-study group was mainly a result of improvement in sensitivity for T1b2 staging ( $80.3 \%$ in the self-study group versus $65.8 \%$ in the non-selfstudy group). Clinically, this effect can be expected to reduce excessive ESD, which may result in additional gastrectomy with lymph node dissection, by 14.5 percentage points by reducing underestimation of invasion depth of EGCs. In this study, however, specificity for T1b2 staging (i. e., the ability to correctly exclude the T1a-T1b1 lesions) in the self-study group $(80.3 \%)$ was lower than in the non-self-study group (85.8\%), which may, in turn, increase the possibility of over-surgery. Tsujii et al. reported that EUS could salvage $60 \%$ of the T1a-T1b1 lesions that are over-diagnosed as T1b2 by conventional WLE [5]. Practically, we therefore recommend adding EUS evaluation of lesions positive for non-extension sign to avoid over-surgery. 
To clarify the limitation of depth prediction using the nonextension sign, we compared correct T-staging rates between shallow T1b2 lesions $(500-999 \mu \mathrm{m})$ and deep T1b2 lesions ( $\geq$ $1000 \mu \mathrm{m})$. The results revealed that correct T-staging rate was significantly lower in shallow T1b2 lesions even after the participants were exposed to our e-learning programs. Identification of minute non-extension sign in those shallow T1b2 lesions might be rather difficult.

The most important point when assessing presence of the non-extension sign in a target EGC is observation of the lesion from an oblique position with sufficient extension of the gastric wall ( $\triangleright$ Fig. 3b). When we observe the lesion from the frontal view, mucosal elevation made by invasive cancer cells is difficult to recognize ( $\boldsymbol{F}$ Fig. $\mathbf{3 a}$ ), which may lead to the underestimation of invasion depth. And when we observe the lesion with a small amount of air in the stomach, surface irregularity or margin elevation can be excessively emphasized, which may lead to the overestimation of invasion depth. As we show in - Fig. 3 , in some cases in the diagnostic test, we intentionally included one endoscopic photo taken with a frontal view or with insufficient air volume. The correct answer rates in such cases were generally low in the pretest, but improved over 20 percentage points in post-test 2 after the participants understood the appropriate conditions under which to evaluate the non-extension sign in our e-learning program (results are not shown).

An e-learning program is now recognized as a useful educational tool in the health professions, and how to construct effective learning content is a matter of interest [9]. Before starting this trial, we had already developed an e-learning program for how to detect EGC with WLE $[10,11]$ and how to differentiate EGC from benign lesions using magnifying narrow band imaging (NBI) [12]. Both e-learning programs were proven in large randomized controlled trials to be useful for endoscopists to acquire diagnostic knowledge. In those trials, because we provided both video lectures and self-study quizzes, how either of the contents accounted for the participants' improvement was unclear. However, in the current study, the educational effect of the self-study was clarified as reinforcement of acquired knowledge. We also found that the learning effect was reduced when endoscopists did not accumulate experience by continuing the practice using the self-study quizzes.

There were a few limitations to this study. First, we did not evaluate improvement in diagnostic accuracy in actual clinical practice. In the diagnostic test, we did not include lesions in which the non-extension sign was not effective for T-staging (i. e., false-positive lesions, false-negative lesions, or low-quality images). Improvement in test score, therefore, might not directly reflect improvement in diagnostic accuracy in the real clinical setting. We need to validate the effectiveness of this elearning program in real clinical practice. Second, two cases of EGC that that had already been used in the previous report [2] were unintentionally included in our diagnostic test. A few participants might have been aware of the fact; however, considering the large number of participants $(>400)$ in this randomized controlled trial, we assume it did not affect our results greatly. Third, we constructed this e-learning program in Japanese and did not invite endoscopists outside Japan. To generalize the results of our study, an international trial using an English version of the e-learning program is required.

\section{Conclusion}

In conclusion, our e-learning program for the non-extension sign that comprised video lectures and self-study quizzes, supplying both knowledge and experience, improved endoscopists' ability to diagnose invasion depth of EGC. We believe that this concept may be applicable to education for various types of endoscopic diagnoses.

\section{Acknowledgements}

This research was supported by a research grant from Central Research Institute for Endoscopy, Fukuoka University. The authors thank Ms. Ryoko Yanagida and Ms. Asuka Mizushima (Department of Molecular-Targeting Cancer Prevention, Kyoto Prefectural University of Medicine) for their assistance with data management. We are sincerely grateful to Mr. Yuichi Takahashi (FAST Inc., Tokyo, Japan) for his engineering work to construct the website and the e-learning system used in this study. We thank Cathel Kerr, PhD, from Edanz Group (www.edanzediting. com/ac) for editing a draft of this manuscript.

\section{Competing interests}

None

\section{References}

[1] Japanese Gastric Cancer Association. Japanese gastric cancer treatment guidelines 2014 (ver. 4). Gastric Cancer 2017; 20: 1 - 9

[2] Nagahama T, Yao K, Imamura K et al. Diagnostic performance of conventional endoscopy in the identification of submucosal invasion by early gastric cancer: the "non-extension sign" as a simple diagnostic marker. Gastric Cancer 2017; 20: 304-313

[3] Abe S, Oda I, Shimazu T et al. Depth-predicting score for differentiated early gastric cancer. Gastric Cancer 2011; 14: 35-40

[4] Sano T, Okuyama Y, Kobori O et al. Early gastric cancer endoscopic diagnosis of depth of invasion. Dig Dis Sci 1990; 35: $1340-1344$

[5] Tsujii Y, Kato M, Inoue T et al. Integrated diagnostic strategy for the invasion depth of early gastric cancer by conventional endoscopy and EUS. Gastrointest Endosc 2015; 82: 452 - 459

[6] Choi J, Kim SG, Im JP et al. Comparison of endoscopic ultrasonography and conventional endoscopy for prediction of depth of tumor invasion in early gastric cancer. Endoscopy 2010; 42: 705 - 713

[7] Yanai H, Noguchi T, Mizumachi S et al. A blind comparison of the effectiveness of endoscopic ultrasonography and endoscopy in staging early gastric cancer. Gut 1999; 44: $361-365$

[8] Choi J, Kim SG, Im JP et al. Endoscopic prediction of tumor invasion depth in early gastric cancer. Gastrointest Endosc 2011; 73: 917 - 927

[9] Pimentel-Nunes P, Buxbaum J. Internet based e-learning systems: a tool for the future in endoscopy. Endoscopy 2017; 49: 936 -937 
[10] Yao K, Uedo N, Muto M et al. Development of an E-learning system for the diagnosis of early gastric cancer: an international multicenter randomized controlled trial. EBioMedicine 2016; 9: 140 - 147

[11] Yao K, Uedo N, Muto M et al. Development of an e-learning system for teaching endoscopists how to diagnose early gastric cancer: basic principles for improving early detetion. Gastric Cancer 2017; 20 : $\mathrm{S} 28-\mathrm{S} 38$

[12] Nakanishi $\mathrm{H}$, Doyama $\mathrm{H}$, Ishikawa $\mathrm{H}$ et al. Evaluation of an e-learning system for diagnosis of gastric lesions using magnifying narrow-band imaging: a multicenter randomized controlled study. Endoscopy 2017; 49: $957-967$ 